\title{
Caracterización morfológica de hongos asociados al agroecosistema café (Coffea arabica L.), en el estado de Tabasco, México
}

\section{Morphological characterization of fungi associated with the coffee agroecosystem (Coffea arabica L.), in the state of Tabasco, Mexico}

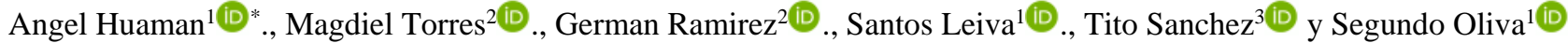

\section{RESUMEN}

El objetivo del trabajo fue identificar a nivel morfológico los hongos asociados al agroecosistema café (Coffea arabica L.), en el estado de Tabasco, México. En campo se seleccionaron al azar 6 plantas de café y se colectaron muestras de hojas tallos y frutos con síntomas visuales atribuidos a hongos. En laboratorio se realizaron aislamientos en medio PDA a partir del tejido enfermo y se obtuvieron cepas puras. Para la caracterización morfológica a nivel de género se emplearon claves especializas (forma, color y tamaño). En total se obtuvieron 47 aislamientos, los cuales fueron distribuidos por órganos de la planta: en hojas hubo 22 aislados con afinidad morfológica con Colletotrichum gloesporoides, tres con Colletotrichum theobromicola, uno con Pestalotiopsis sp, tres con Paramyrothecium roridum, uno con Phomopsis sp, dos Hemileia vastatrix y dos con Marasmius sp; en el cuello de la planta se encontró un Fusarium sp; en el tallo Lasiodiplodia sp y un Colletotrichum gigasporum; en frutos se reportó nueve aislados de Fusarium sp. Se identificaron 11 especies de hongos, asociados al patosistema Coffea arabica L., en sus diversos órganos; de éstos, 10 están reportadas como fitopatógenos este cultivo y uno como patógeno de Theobroma cacao L.

Palabras clave: Aislamiento fúngico, ascomyceto, caficultura ecológica, Marasmius sp., patógeno.

\begin{abstract}
The objective of this work was to identify at morphological level the fungi associated with the coffee agroecosystem (Coffea arabica L.), in the state of Tabasco, Mexico. In the field, 6 coffee plants were randomly selected and samples of leaves, stems and fruits with visual symptoms attributed to fungi were collected. In the laboratory, isolations were made on PDA medium from the diseased tissue and pure strains were obtained. For morphological characterisation at genus level, specialised keys (shape, colour and size) were used. A total of 47 isolates were obtained, which were distributed by plant organ: in leaves there were 22 isolates with morphological affinity with Colletotrichum gloesporoides, three with Colletotrichum theobromicola, one with Pestalotiopsis sp, three with Paramyrothecium roridum, one with Phomopsis sp, two with Hemileia vastatrix and two with Marasmius sp; one Fusarium sp was found on the collar of the plant; Lasiodiplodia sp and one Colletotrichum gigasporum were found on the stem; nine isolates of Fusarium sp were reported on the fruit. Eleven species of fungi associated with the pathosystem Coffea arabica L., in its various organs; of these, 10 are reported as phytopathogens of this crop and one as a pathogen of Theobroma cacao L.
\end{abstract}

Keywords: Fungal isolation, ascomycete, ecological coffee farming, Marasmius sp., pathogen.

DOI: https://doi.org/10.37787/pakamuros-unj.v9i3.217

Recibido: 06/05/2021. Aceptado: 08/07/2021

* Autor para correspondencia

1. Instituto de Investigación para el Desarrollo Sustentable de Ceja de Selva, Universidad Nacional Toribio Rodríguez de Mendoza de Amazonas, calle Higos Urco N 342-350-356 - Calle Universitaria N 304. Perú. Email: angel.huaman@untrm.edu.pe ; santos.leiva@untrm.edu.pe ; soliva@indes-ces.edu.pe

2. División Académica de Ciencias Biológicas, Universidad Juárez Autónoma de Tabasco, carretera Villahermosa Cárdenas km 0.5, CP 86039, México. Email: biomag75@hotmail.com; yerman_122@hotmail.com

3. Instituto de Investigaciones de la Amazonia Peruana, Av. Abelardo Quiñones Km 2.5 Iquitos-Loreto, Perú. Email: titosancheazsantillan@gmail.com 


\section{INTRODUCCIÓN}

El origen del café en su forma silvestre conocida como Arabica es atribuido al altiplano de Abisinia (actual Etiopia), existe una serie de leyendas respecto a su descubrimiento como bebida. La leyenda más aceptada hace a Kaldi el pastor quien observó que sus cabras mostraban un comportamiento diferente a las demás, estas se veían llenas de energía después de haber comido las hojas y frutos de cierto arbusto, posteriormente comenzó a cultivar estas plantas (Gotteland \& De Pablo, 2007). El cultivo de café es una actividad económica importante para los ingresos económicos de caficultores en diferentes países en el mundo, la gran demanda mundial del grano del café, ubica a este como el segundo producto de mayor exportación solo después del petróleo (DaMatta et al., 2007). En México existe 12 entidades federativas con mayor actividad económica cafetalera, que son: Chiapas, Colima, Guerrero, Hidalgo, Jalisco, Nayarit, Oaxaca, Puebla, Querétaro, San Luis Potosí, Tabasco y Veracruz (Vichi, 2015), el estado de Tabasco tiene 1006 ha aproximadamente de café, sin embargo, cuenta con amplia área con características idóneas para el cultivo de café (Aceves Navarro et al., 2018).

Los caficultores están bajo una constante amenaza de problemas fitopatológicos, muchas de estas están como potenciales a convertirse en problemas claves a futuro, tales como; el ojo de gallo Mycena citricolor, mal de hilachas Pellicularia koleroga, antracnosis Colletotrichum gloesporoides, la pudrición negra de la raíz Rosellinia bunodes, nematodos, también se tiene a patógenos e insectos causando grandes pérdidas económicas como la roya (Hemileia vastatrix) y barrenador de fruto (Hipothenemus hampei) que afectan el rendimiento y la calidad del café (Rutherford \& Phiri, 2006). Por otro lado, se están reportando nuevas especies patogénicas asociados al cultivo de café, como muerte del café (Lasidoplodia pseudothebromae), en Brasil (Freitas-Lopes et al., 2020), mancha foliar del café (Paramyrothecium roridum), actualmente considerado como amenaza para los viveros en México (Pelayo-Sánchez et al., 2017), en el sur de China se reportó dos especies de pestalotiopsis (Pestalotiopsis coffeae-arabicae y Pestalotiopsis rhodomyrtus), los síntomas de esta especie se muestran como manchas cloróticas de color marron-rojizo en las hojas (Song et al., 2013), así mismo (Gong et al., 2020), en China, realizó el primer reporte del tizón de la hoja del café Phomopsis heveicola (anamorfico de Diaporthe tulliensis).

En México existen áreas de producción cafetalera en crecimiento, pero hay escasa evidencia científica de identificación y caracterización de enfermedades fitopatogenas asociados al cultivo de café, que es información clave para el manejo integrado de plagas y enfermedades de este cultivo. 
Bajo este contexto nuestra investigación hongos asociados al patosistema café (Coffea arabica L.), busca generar investigación básica para futuras investigaciones, para lo cual, se realizó muestreos de 3 municipios cafetaleros del estado de Tabasco, se realizaron los aislamientos a nivel monospóricos en el laboratorio de Entomopatógenos de la Universidad Juárez Autónoma de Tabasco - UJAT, se realizó la caracterización macroscópica y microscópica de 47 cepas.

\section{MATERIALES Y MÉTODOS}

\section{Descripción del área de estudio}

Los sitios de muestreo se ubicaron en tres municipios que concentran la producción de café en el área: Teapa, Huimanguillo y Tacotalpa. En cada municipio se establecieron 2 sitios de muestreo (Tabla 1). El muestro se realizó en los meses de enero y febrero de 2020. En cada parcela se recolectaron hojas, frutos y tallos con síntomas de enfermedades fitopatógenas y se trasladaron para su aislamiento, al laboratorio de sanidad vegetal de la Universidad Juárez Autónoma de Tabasco.

Tabla 1. Sitios de muestreo y aislamientos de hongos obtenidos a tejidos vegetales sintomático en plantaciones de café de Tabasco, México.

\begin{tabular}{|c|c|c|c|c|c|}
\hline $\begin{array}{l}\text { Municipio } \\
\text { Teapa }\end{array}$ & \multirow{2}{*}{$\begin{array}{l}\text { Localidad } \\
\text { Nicolas } \\
\text { Bravo } 1\end{array}$} & \multicolumn{2}{|c|}{ Georreferenciación } & \multirow{2}{*}{$\begin{array}{c}\begin{array}{c}\text { Altitud } \\
\text { (m.s.n.m.) }\end{array} \\
423\end{array}$} & \multirow{2}{*}{$\begin{array}{l}\begin{array}{c}\text { Aislamientos } \\
\text { obtenidos }\end{array} \\
\text { cpt1, cpt2, cpt3, } \\
\text { cpt4, cpt5, cpt6, } \\
\text { cpt7, cpt8, cpt9, } \\
\text { cpt10 }\end{array}$} \\
\hline Teapa & & N17³0'55.6" & W09257'17.6" & & \\
\hline & $\begin{array}{l}\text { Nicolas } \\
\text { Bravo2 }\end{array}$ & N17²9'46.1" & W09257'26.9" & 473 & $\begin{array}{l}\text { cpt11, cpt12, } \\
\text { cpt13, cpt13, } \\
\text { cpt15 }\end{array}$ \\
\hline \multirow[t]{2}{*}{ Huimanguillo } & Jmujica & N17²0'21.6" & W09336'03.6" & 407 & $\begin{array}{l}\text { cpt16, cpt17, } \\
\text { cpt18, cpt19, } \\
\text { cpt20, cpt21, } \\
\text { cpt22, cpt23, } \\
\text { cpt24, cpt25, } \\
\text { cpt27, cpt26 }\end{array}$ \\
\hline & Malpasito & N1720'21.6" & W094³6'03.6" & 145 & $\begin{array}{l}\text { cpt28, cpt29, } \\
\text { cpt30 }\end{array}$ \\
\hline \multirow[t]{2}{*}{ Tacotalpa } & $\begin{array}{l}\text { Palo } \\
\text { quemado }\end{array}$ & N17²5'07.0" & W09250'09.7" & 956 & $\begin{array}{l}\text { cpt31, cpt32, } \\
\text { cpt33, cpt34, } \\
\text { cpt35, cpt36, } \\
\text { cpt37, cpt38, } \\
\text { cpt39, cpt40 }\end{array}$ \\
\hline & $\begin{array}{l}\text { Cerro } \\
\text { Blanco 4ta }\end{array}$ & N17²5'59.8" & W092 $48^{\prime} 52.6^{\prime \prime}$ & 456 & $\begin{array}{l}\text { cpt41, сpt42, } \\
\text { cpt43, cpt44, } \\
\text { cpt45, cpt46, } \\
\text { cpt47 }\end{array}$ \\
\hline
\end{tabular}

CPT: Cepa Patosistema Tabasco; clave de laboratorio asignada a los aislamientos 


\section{Aislamiento de hongos}

Con un bisturí previamente desinfectado, se cortaron pequeños fragmentos de tejido vegetal afectado por el patógeno y se enjuagaron con agua destilada estéril. Posteriormente los fragmentos de muestra se desinfectaron con hipoclorito de sodio al $2 \%$ por dos minutos. Las muestras se enjuagaron dos veces con agua destilada estéril, con el propósito de quitar el sobrante de hipoclorito de sodio. Consecuentemente, bajo condiciones asépticas, se distribuyeron 5 fragmentos del tejido enfermo en cajas Petri conteniendo medio de cultivo papa dextrosa agar (PDA). Se incubaron de cinco a siete días, tiempo necesario para el desarrollo del micelio. Posteriormente se transfirió una fracción del medio de cultivo con el micelio a una caja Petri nueva con media de cultivo PDA. Finalmente, de cada aislamiento poliespórico se obtuvieron los aislamientos monospóricos (Estrada et al., 1997).

\section{Identificación morfológica}

Las especies de hongos se identificaron teniendo como base a estructuras reproductivas (conidióforos, fiálides, conidios y clamidosporas), de las cuales se colectaron datos sobre descripciones morfológicas (forma, color y tamaño), descrita por autores mencionados; para Colletotrichum (Cristóbal-Martínez et al., 2017; James et al., 2014, 2014; Nguyen et al., 2010; Prihastuti et al., 2009; Rojo-Báez et al., 2017; Singh \& Doyle, 2017; Weir et al., 2012), para Fusarium (Nalim et al., 2011; Nguyen et al., 2010; Robles Yerena et al., 2016; Summerell et al., 2011; Weir et al., 2012); para Lasidoplodia (Freitas-Lopes et al., 2020; Liang et al., 2019); para Marasmius (Amoako-Attah et al., 2016); para Paramyrothecium (Da Silveira et al., 2007; Pelayo-Sánchez et al., 2017); para Pestalotiopsis (Song et al., 2013); para Phomopsis (Gong et al., 2020; Mahadevakumar et al., 2017); para Hemileia (Carvalho et al., 2011). Para esto, los aislamientos se desarrollaron en caja petri conteniendo medio PDA durante 7 días y facilite describir dichas características macroscópicas. Así mismo, se realizó microcultivos para obtener estructuras microscópicas de cada aislamiento. Todos los microcultivos se incubaron a $25^{\circ} \mathrm{C}$ en cámara húmeda durante 3 días. Transcurrido este tiempo, se observaron las características microscópicas (conidioros, conidios, apresorium y clamidisporas), examinados bajo el microscopio de campo claro, éstas a su vez se fotodocumentaron mediante digitalización de imágenes y posterior morfometría con el software Image Tool® 3.00 y ZEN. 


\section{RESULTADOS}

Se obtuvieron 47 aislamientos a partir de muestras de tejidos con síntomas de hongos asociados a $C$. arabica en Tabasco, México (Tabla 2).

Tabla 2. Descripción morfológica hongos aislados de plantas de café (C. arabica) de Tabasco, México.

\begin{tabular}{|c|c|c|c|}
\hline Especies & Descripción de colonia & Conidios, esporas & Aislados \\
\hline $\begin{array}{l}\text { Colletotrichum } \\
\text { gloesporoides }\end{array}$ & $\begin{array}{l}\text { Color blanco-cremoso tornándose } \\
\text { grisáceo posterior a los } 7 \text { días; El } \\
\text { reverso de coloración obscura a partir } \\
\text { del centro tornándose clara hacia la } \\
\text { orilla de la caja petri. }\end{array}$ & $\begin{array}{l}\text { Conidios } \\
\text { L: } 11.70-15.91 \mu \mathrm{m} \\
\text { A: } 3.89-5.26 \mu \mathrm{m}\end{array}$ & $\begin{array}{l}\text { СРТ2, СРТ6, СРТ9, СРТ10, } \\
\text { СРТ11, СРТ12, СРТ13, } \\
\text { СРТ15, СРТ17, СРТ25, } \\
\text { СРТ31, СРТ28, СРТ19, } \\
\text { СРТ23, СРТ25, СРТ28, } \\
\text { СРТ31, СРТ33, СРТ35, } \\
\text { СРТ37, СРT40, СРТ41 }\end{array}$ \\
\hline $\begin{array}{l}\text { Colletotrichum } \\
\text { theobromicola }\end{array}$ & $\begin{array}{l}\text { Abundante micelio aéreo de } \\
\text { coloración grisácea que luego de los } \\
\text { siete días se fue tornando más } \\
\text { oscuro; Al reverso se mostró un color } \\
\text { negro intenso en el centro, } \\
\text { tornándose gris hacía los extremos. }\end{array}$ & $\begin{array}{l}\text { Conidios } \\
\text { L: } 12.95-18.32 \mu \mathrm{m} \\
\text { A: } 4.06-5.22 \mu \mathrm{m}\end{array}$ & СРТ7, СРТ16, СРТ21 \\
\hline $\begin{array}{l}\text { Colletotrichum } \\
\text { gigasporum }\end{array}$ & $\begin{array}{l}\text { Abundante micelio aéreo algodonoso } \\
\text { de color gris, márgenes de color } \\
\text { blanquecino, pero se tornan gris y } \\
\text { aparecen abundantes acervulus en la } \\
\text { periferia de la colonia con el avance } \\
\text { de los días; El reverso de la placa de } \\
\text { observó de color grisáceo con puntos } \\
\text { de color negro que forman los } \\
\text { acervulus. }\end{array}$ & $\begin{array}{l}\text { Conidios } \\
\text { L: } 20.00-32.29 \mu \mathrm{m} \\
\text { A: } 8.07-11.9 \mu \mathrm{m}\end{array}$ & СРТ 8 \\
\hline Fusarium solani & $\begin{array}{l}\text { Coloración blanco-cremoso- } \\
\text { amarillento de aspecto afelpado con } \\
\text { micelio aéreo fino formando anillos } \\
\text { concéntricos; El reverso se muestra } \\
\text { un color amarillo-oscuro. }\end{array}$ & $\begin{array}{l}\text { Macroconidios } \\
\text { L: } 30.65-54.53 \\
\text { A: } 4.02-5.52 \mu \mathrm{m} \\
\text { Microconidios } \\
\text { L: } 7.08-14.12 \mu \mathrm{m} \\
\text { A: } 2.12-5.24 \mu \mathrm{m}\end{array}$ & $\begin{array}{l}\text { СРТ3, СРТ4, СРТ5, СРТ14, } \\
\text { СРТ18, СРТ24, СРТ29, } \\
\text { СРТ30, СРТ39 }\end{array}$ \\
\hline Fusarium sp & $\begin{array}{l}\text { Aspecto algodonoso y de color } \\
\text { blanquecino al inicio, rojizo con el } \\
\text { avance de los días; El reverso se } \\
\text { muestra un color rojizo oscuro en el } \\
\text { centro con color blanquecino a los } \\
\text { extremos. }\end{array}$ & $\begin{array}{l}\text { Microconios } \\
\text { L: } 5.10-11.90 \mu \mathrm{m} \\
\text { A: } 1.50-2.70 \mu \mathrm{m}\end{array}$ & СРТ26 \\
\hline
\end{tabular}




\begin{tabular}{|c|c|c|c|}
\hline Pestalotiopsis sp & $\begin{array}{l}\text { El micelio aéreo de color } \\
\text { blanquecino, borde ondulado, con el } \\
\text { avance de los días aparecieron } \\
\text { cuerpos fructíferos de color negro en } \\
\text { el micelio; El reverso de la caja } \\
\text { presento una coloración blanca a } \\
\text { amarillo pálido }\end{array}$ & $\begin{array}{l}\text { Conidios } \\
\text { L: } 19.83-23.92 \mu \mathrm{m} \\
\text { A: } 5.69-8.11 \mu \mathrm{m} \\
\text { Apéndices apicalesL: } \\
11.20-22.70 \mu \mathrm{m} \\
\text { Apéndice basal } \\
\text { L: } 4.40-7.60 \mu \mathrm{m}\end{array}$ & CPT1 \\
\hline $\begin{array}{l}\text { Lasiodiplodia } \\
\text { pseudotheobromae }\end{array}$ & $\begin{array}{l}\text { Abundante micelio aéreo de color } \\
\text { blanco al inicio, tornándose grisáceo } \\
\text { con el avance de los días; El reverso } \\
\text { de la colonia mostro una coloración } \\
\text { gris }\end{array}$ & $\begin{array}{l}\text { Conidios } \\
\text { L: } 21.52-31.22 \\
\text { A: } 11.75-18.66\end{array}$ & СРТ22, СРТ34 \\
\hline $\begin{array}{l}\text { Paramyrothecium } \\
\text { roridum }\end{array}$ & $\begin{array}{l}\text { Micelio aéreo de color blanco y } \\
\text { formando anillos concéntricos que } \\
\text { contenían abundantes esporodoquios } \\
\text { de color verde-oliváceo; El reverso } \\
\text { de la colonia se observa de color } \\
\text { amarillo pálido, se muestran los } \\
\text { anillos concéntricos }\end{array}$ & $\begin{array}{l}\text { Conidios } \\
\mathrm{L}: 5.23-6.75 \mu \mathrm{m} \\
\mathrm{A}: 1.37-1.99 \mu \mathrm{m}\end{array}$ & СРТ36, СРТ38 у СРТ42 \\
\hline Phomopsis sp & $\begin{array}{l}\text { Color blanquecino con aspecto } \\
\text { algodonoso y margen ondulado, } \\
\text { presentan cuerpos fructíferos de } \\
\text { color marrón dispersos o en grupos } \\
\text { en el micelio; El reverso de la caja } \\
\text { Petri fue de color blanquecino en los } \\
\text { primeros días tornándose color gris } \\
\text { pálido luego }\end{array}$ & $\begin{array}{l}\text { Beta-conidios } \\
\text { L: } 5.80-8.80 \mu \mathrm{m} \\
\text { A: } 1.60-3.10 \mu \mathrm{m} \\
\text { Alfa-conidios } \\
\text { A: } 18.80-43.20 \\
\text { L: } 0.90-1.50\end{array}$ & СРТ32 \\
\hline Marasmius sp & $\begin{array}{l}\text { Crecimiento con abundante micelio } \\
\text { en forma de hilo, de color blanco } \\
\text { algodonoso }\end{array}$ & $\begin{array}{l}\text { No forma conidios en } \\
\text { PDA }\end{array}$ & СРТ 44, СРТ 47 \\
\hline Hemileia vastatrix & Parasito obligado & $\begin{array}{l}\text { Uredosporas } \\
\mathrm{L}: 28-34.3 \mu \mathrm{m} \\
\mathrm{A}: 18-28 \mu \mathrm{m}\end{array}$ & СРТ46, СРТ47 \\
\hline
\end{tabular}

L: Largo; A: Ancho

De los aislamientos obtenidos, 22 presentaron afinidad morfológica con Colletotrichum gloesporoides, tres con Colletotrichum theobromicola, uno con Colletotrichum gigasporum, nueve con Fusarium solani, uno con Fusarium sp, uno con Pestalotiopsis sp, dos con Lasiodiplodia pseudotheobromae, tres con Paramyrothecium roridum, uno con Phomopsis sp, dos con Marasmius sp. y dos con Hemileia vastatrix; dichas muestras comparadas correspondió a las capturas fotográficas de los conidióforos, conidios y apresorios de los hongos (Figura 1, 2, 3 y 4). 


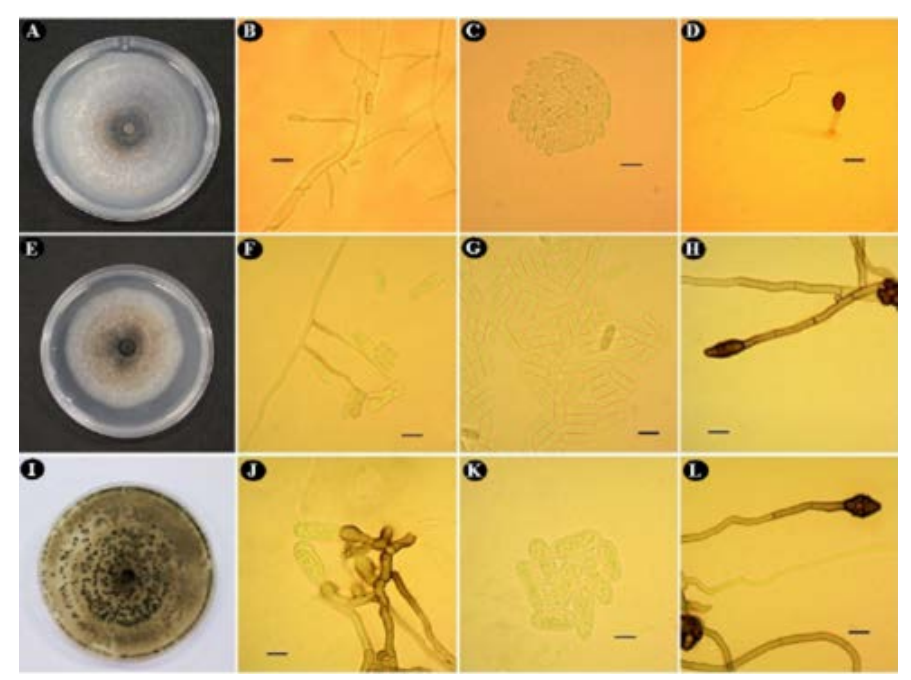

Figura 1. Hongos asociados al agroecosistema de Coffea arabica, en medio PDA, a $25^{\circ}$ C. A. Colonia de C.gloesporoides; B, C y D. Conidióforo, conidios y apresorios de C.gloesporoides respectivamente. E. Colonia de C.theobromicola; F, G y H. Conidióforo, conidios y apresorios de C.theobromicola; I. Colonia de C.gigasporum; J, K y L. Conidióforo, conidios y apresorios de C.gloesporoides respectivamente. Barra 10 um

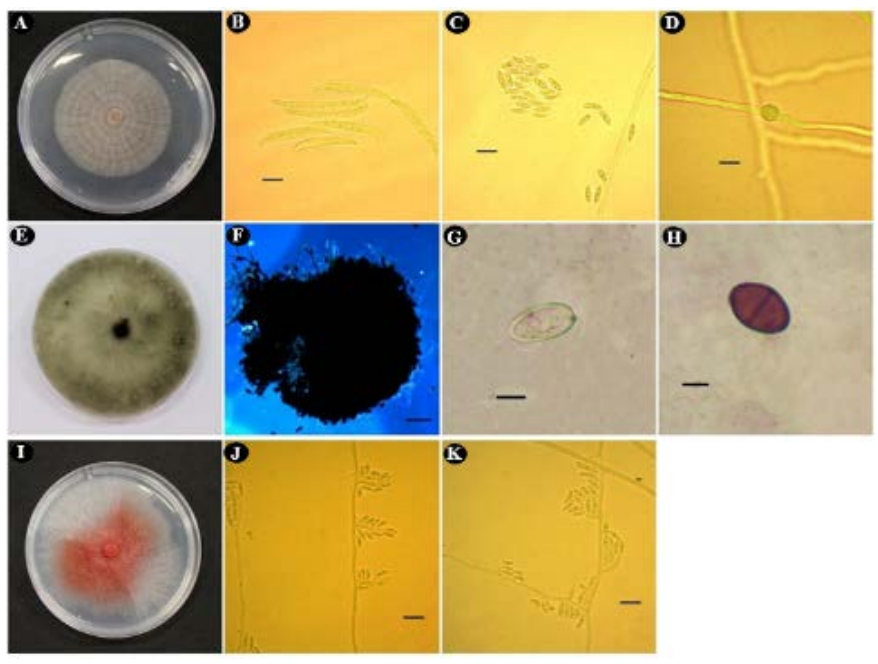

Figura 2. Hongos de los géneros Fusarium y Lasiodiplodia, asociados al agroecosistema Coffea arabica, en medio de cultivo PDA. A. Colonia de F.solani; B, C y D. Macronidios, micronidios y clamidospora de F.solani respectivamente; E. Colonia de L.pseudotheobromae; F, G y H. Picnidio, Conidio inmaduro y conidio maduro de L.pseudotheobromae respectivamente; I. Colonia de Fusarium sp; J y K. Conidióforos y micronidios de L.pseudotheobromae respectivamente. Barra $10 \mathrm{um}$. 

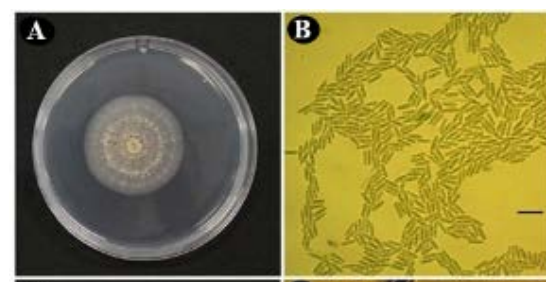

C
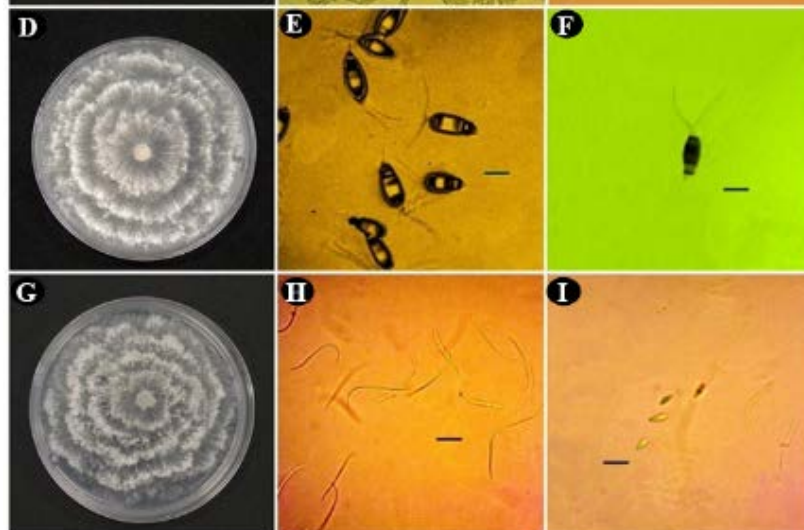

(1)

Figura 3 A. Colonia de P.roridum, B y C. Conidios y conidióforos de P.roridum respectivamente D. Colonia de Pestalotiopsis sp; E y F. Conidios con un apéndice basal y dos apéndices apicales G. Colonia de Phompsis sp; H y I. Beta-conidios y alfa-conidios de Phompsis sp respectivamente. Barra 10 um.

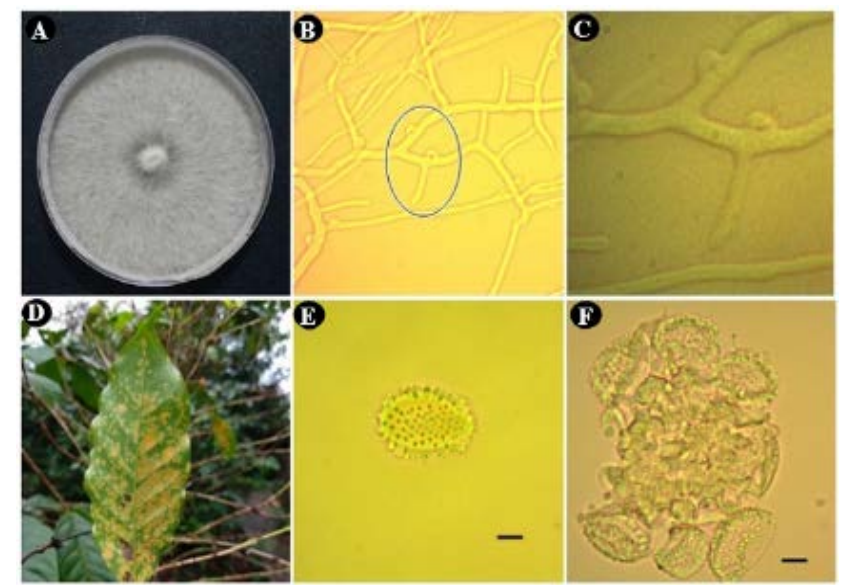

Figura 4. A. Colonia de Marasmius sp; B y C. Conexiones tipo asa o clamp conection de las hifas; D. Hoja de café mostrando síntomas de H.vastratrix; E y F. Uredospora y uredio de H.vastatrix respectivamente 


\section{DISCUSIÓN}

Se evaluó la diversidad de hongos asociados al cultivo de C. arabica en Tabasco, México. Si bien, existe escasa información o reportes de la diversidad de hongos asociados al patosistema café en este lugar. Como parte del estudio, para la identificación de estos patógenos, requiere fundamentalmente describir sus rasgos morfológicos (forma de cuerpos fructíferos, micelio, conidiación, crecimiento en medio de cultivo, entre otros) (Saucedo-García et al., 2014). En efecto, en esta investigación se partió por el estudio de las características morfológicas de los patosistemas; de los cuales se identificaron 11 especies. A pesar de los esfuerzos y la corroboración con otros trabajos de la existencia de estos patógenos, el estudio crea precedentes para futuros estudios ya sea nivel morfológico y molecular.

Iniciando con identificación de Colletotrichum, en la presente investigación, Cristóbal-Martínez et al. (2017), ya reportaron la existencia de 46 especies de Colletotrichum en plantaciones de café, que fueron distribuidas en C. theobromicola, C. gloesporoides y C. gigasporum. Por su parte, Prihastuti et al. (2009), realizaron caracterización de especies de Colletotrichum en el norte de Tailandia, encontrando Colletotrichum gloesporoides que estuvieron específicamente en frutos de café; similar reportaron Nguyen et al. (2010), especies de Colletotrichum gloesporoides presentes frutos y hojas de café; guardando relación a lo encontrado en la presente investigación, tal parece que esta especie afecta más en órganos específicos.

A partir de frutos, se identificaron especies de Fusarium solani, no obstante, aún no se han reportado la presencia de este patógeno en este órgano de planta. En otros estudios, Torres et al. (2019), reportaron especies Fusarium solani, asociados con Hipotenemus hampei, lo cual, hace indicar que es pisble el atque del patógno a este organo de la planta, por una relación analófgica de ataques con dicha plaga. Esto es corroborado por Nalim et al. (2011), en la que datan que Fusarium solani, posee una amplia gama de hospederos causandolas severeos daños. Fusarium sp, también fueron encontrados en la investigación a partir de sintomas de pudricion del cuello de la plantula, por lo que, López-Lima et al. (2020), afirma que Fusarium osysporum, es un complejo de hongos más importantes que causa la marchitez de plantas especificamente en café.

Otra especie encontrada fue Lasiodiplodia pseudotheobromae mencionada como primer registro en el patosistema café en Tabasco; para el café, esta especie ya fue reportada y por primera vez en Brasil (Freitas-Lopes et al. (2020); también en Tailandia y en café (Trakunyingcharoen et al., 2015). 
En hojas se encontraron Pestalotiopsis sp, caracterizando a cada género las células conidiógenas (conidios simples septados de pared lisa con apéndices apicales y una basal), según los reportes hechas por Song et al. (2013), para cultivos de café en el sur de China.

Otra especie patógena importante identificada fue Paramyrothecium roridum; dicha ya ha sido reportada a partir de hojas en café a nivel de vivero en Oaxaca, México (Pelayo-Sánchez et al., 2017). Tambien, fue reportada a nivel de vivero en Río de Janeiro, Barasil, asociadolas como sinomina a Myrothecium roridum (Lombard \& Crous, 2016). No obstante, en la presente investigación se encontraron en plantaciones de café en campo (promedio 2 años de edad).

Un reporte importante se tuvo con el género Phomopsis, que normalmente fueron reportados en leguminosas (Brumer et al., 2018), no obstante, también atacan al cafeto; siendo, reportado por primera vez en China, a partir de hojas, que causaban de $20 \%$ a $30 \%$ en promedio de incidencia (Gong et al., 2020).

Marasmius sp fue otra especie encontrada en hojas (mal de hilachas); para café, aún se desconoce reportes de este género. No obstante, ya fue reportada la especie Marasmius scandens, en Theobroma cacao, con el cual se comparó el hongo asilado en la investigación y que generaron síntomas similares (Amoako-Attah et al., 2016).

Finalmente, se ha encontrado en mayor cantidad especies de Hemileia vastatrix, en todas las zonas muestreadas, con baja incidencia de daño. No cabe duda que, $H$. vastatrix es patógeno con mayor ataque en plantas de café y además que posse una amplia variabilidad genetica (Malau, 2019; Quispe-Apaza et al., 2017; Talhinhas et al., 2017).

\section{CONCLUSIONES}

Se identificaron 11 especies de hongos, asociados al patosistema C. arabica L., en sus diversos órganos; de éstos, 10 están reportadas como fitopatógenos este cultivo y uno como patógeno de T. cacao L., mediante el aislamiento y forfología cualitativa (forma y color) y cuantitativa (tamaño). En hojas se idenificaron (C.gloesporides, C.theobromicola, Pestalotiopsis sp, Paramyrothecium roridum, Phomopsis sp y Hemileia vastatrix), en el cuello de plátulas con pudriciones (Fusarium sp), en frutos (Lasiodiplodia pseudotheobromae y C. gigasporum) y en tallos (Fusarium solani). Adicionalmente se identificó Marasmius sp. en hojas, sin embargo, no se encuentra registrado como patógeno de C. arabica. 


\section{REFERENCIAS BIBLIOGRÁFICAS}

Aceves Navarro, L. A., Rivera Hernández, B., López Castañeda, A., Palma López, D. J., González Mancillas, R., \& Juárez López, J. F. (2018). Áreas potenciales y vulnerabilidad del cultivo de café tipo robusta (Coffea canephora P.) al cambio climático en el estado de Tabasco, México. Nova scientia, 10(20), 369-396. https://doi.org/10.21640/ns.v10i20.1379

Amoako-Attah, I., Akrofi, A. Y., Hakeem, R. B., Asamoah, M., \& Kumi-Asare, E. (2016). White thread blight disease caused by Marasmiellus scandens (Massee) Dennis Reid on cocoa and its control in Ghana. African Journal of Agricultural Research, 11(50), 5064-5070. https://doi.org/10.5897/AJAR2016.11681

Brumer, B. B., Lopes-Caitar, V. S., Chicowski, A. S., Beloti, J. D., Castanho, F. M., da Silva, D. C. G., de Carvalho, S., Lopes, I. O., Soares, R. M., \& Seixas, C. D. (2018). Morphological and molecular characterization of Diaporthe (Phomopsis anamorph) complex and pathogenicity of Diaporthe aspalathi isolates causing stem canker in soybean. European Journal of Plant Pathology, 151(4), 1009-1025.

Carvalho, C. R., Fernandes, R. C., Carvalho, G. M. A., Barreto, R. W., \& Evans, H. C. (2011). Cryptosexuality and the genetic diversity paradox in coffee rust, Hemileia vastatrix. Plos one, 6(11), e26387. https://doi.org/10.1371/journal.pone.0026387

Cristóbal-Martínez, A. L., de Jesús Yáñez-Morales, M., Solano-Vidal, R., Segura-León, O., \& Hernández-Anguiano, A. M. (2017). Diversity of Colletotrichum species in coffee (Coffea arabica) plantations in Mexico. European Journal of Plant Pathology, 147(3), 605-614. https://doi.org/10.1007/s10658-016-1029-0

Da Silveira, S. F., Mussi-Dias, V., de Ponte, E. C., \& Dias, P. P. (2007). Myrothecium leaf spot on coffee plants in nursery. Fitopatologia Brasileira, 32(5). https://doi.org/10.1590/S010041582007000500013

DaMatta, F. M., Ronchi, C. P., Maestri, M., \& Barros, R. S. (2007). Ecophysiology of coffee growth and production. Brazilian journal of plant physiology, 19, 485-510. https://doi.org/10.1590/S167704202007000400014

Estrada, M. N., Velez, P. E., \& Lopez, J. C. (1997). Estandarizacion de una metodologia para obtener cultivos monoesporicos del hongo Beauveria bassiana. Centro Nacional de Investigación y Documentación Agropecuaria, (CENIDA), 48(4), 217-224. 
Freitas-Lopes, R. do L., Machado, A. R., \& Lopes, U. P. (2020). Coffee Dieback Caused by Lasiodiplodia pseudotheobromae in Brazil. Plant Disease, 104(3), 980.

Gong, J. L., Lu, Y., Wu, W. H., He, C. P., Liang, Y. Q., Huang, X., Zheng, J. L., Xi, J. G., Tang, S. B., \& Yi, K. X. (2020). First report of Phomopsis heveicola (anamorph of Diaporthe tulliensis) causing leaf blight of Coffee (Coffea arabica) in China. Plant Disease, 104(2), 570. https://doi.org/10.1094/PDIS-09-19-1833-PDN

Gotteland, M., \& De Pablo, S. (2007). Algunas verdades sobre el café. Revista chilena de nutrición, 34(2), 105-115. http://dx.doi.org/10.4067/S0717-75182007000200002

James, R. S., Ray, J., Tan, Y. P., \& Shivas, R. G. (2014). Colletotrichum siamense, C. theobromicola and C. queenslandicum from several plant species and the identification of C. asianum in the Northern Territory, Australia. Australasian Plant Disease Notes, 9(1), 1-6.

Liang, L., Li, H., Zhou, L., \& Chen, F. (2019). Lasiodiplodia pseudotheobromae causes stem canker of Chinese hackberry in China. Journal of Forestry Research, 31(6), 2571-2580. https://doi.org/10.1007/s11676-019-01049-X

López-Lima, D., Carrión, G., Sánchez-Nava, P., Desgarennes, D., \& Villain, L. (2020). Fungal diversity and Fusarium oxysporum pathogenicity associated with coffee corky-root disease in Mexico. Revista De La Facultad De Ciencias Agrarias UNCuyo, 52(1), 276-292.

Mahadevakumar, S., Amruthavalli, C., Sridhar, K. R., \& Janardhana, G. R. (2017). Prevalence, incidence and molecular characterization of Phomopsis vexans (Diaporthe vexans) causing leaf blight and fruit rot disease of brinjal in Karnataka (India). Plant Pathol Quar, 7(1), 29-46. https://doi.org/10.5943/ppq/7/1/5

Malau, S. (2019). Resistance variability of arabica coffee genotypes (Coffea arabica L.) to leaf rust disease (Hemileia vastatrix). Jurnal Tanaman Industri dan Penyegar, 6(2), 69-78. https://doi.org/dx.doi.org/10.21082/jtidp.v6n2.2019.p69-78

Nalim, F. A., Samuels, G. J., Wijesundera, R. L., \& Geiser, D. M. (2011). New species from the Fusarium solani species complex derived from perithecia and soil in the Old World tropics. Mycologia, 103(6), 1302-1330. https://doi.org/10.3852/10-307

Nguyen, P. T. H., Pettersson, O. V., Olsson, P., \& Liljeroth, E. (2010). Identification of Colletotrichum species associated with anthracnose disease of coffee in Vietnam. European Journal of Plant Pathology, 127(1), 73-87. https://doi.org/10.1007/s10658-009-9573-5 
Pelayo-Sánchez, G., Yáñez-Morales, M. J., \& Solano-Vidal, R. (2017). First report of leaf spot on Coffea arabica caused by Paramyrothecium roridum in Mexico. Plant Disease, 101(6), 1044-1044. https://doi.org/10.1094/PDIS-11-16-1588-PDN

Prihastuti, H., Cai, L., Chen, H., McKenzie, E. H. C., \& Hyde, K. D. (2009). Characterization of Colletotrichum species associated with coffee berries in northern Thailand. Fungal Diversity, 39(1), 89-109.

Quispe-Apaza, C. S., Mansilla-Samaniego, R. C., López-Bonilla, C. F., Espejo-Joya, R., VillanuevaCaceda, J., \& Monzón, C. (2017). Genetic diversity of Hemileia vastatrix of two coffee producing areas in Peru. Revista mexicana de fitopatología, 35(3), 418-436. https://doi.org/10.18781/R.MEX.FIT.1612-7

Robles Yerena, L., Leyva Mir, S. G., Cruz Gómez, A., Camacho Tapia, M., Nieto Ángel, D., \& Tovar Pedraza, J. M. (2016). Fusarium oxysporum Schltdl. Y Fusarium solani (Mart.) Sacc. Causantes de la marchitez de plántulas de Pinus spp. En vivero. Revista mexicana de ciencias forestales, 7(36), 25-36. https://doi.org/dx.doi.org/10.21082/jtidp.v6n2.2019.p69-78

Rojo-Báez, I., Álvarez-Rodríguez, B., García-Estrada, R. S., León-Félix, J., Sañudo-Barajas, A., \& Allende-Molar, R. (2017). Situación actual de Colletotrichums spp. en México: Taxonomía, caracterización, patogénesis y control. Revista mexicana de fitopatología, 35(3), 549-570. https://doi.org/10.18781/R.MEX.FIT.1703-9

Rutherford, M. A., \& Phiri, N. (2006). Pests and diseases of coffee in Eastern Africa: A technical and advisory manual. Wallingford, UK: CAB International.

Saucedo-García, A., Anaya, A. L., Espinosa-García, F. J., \& González, M. C. (2014). Diversity and communities of foliar endophytic fungi from different agroecosystems of Coffea arabica L. in two regions of Veracruz, Mexico. PloS one, 9(6), e98454.

Singh, R., \& Doyle, V. P. (2017). Boxwood dieback caused by Colletotrichum theobromicola: A diagnostic guide. Plant Health Progress, 18(3), 174-180. https://doi.org/10.1094/PHP-04-170024-DG

Song, Y., Geng, K., HYDE, K. D., ZHAO, W.-S., Wei, J.-G., Kang, J.-C., \& Wang, Y. (2013). Two new species of Pestalotiopsis from Southern China. Phytotaxa, 126(1), 22-32.

Summerell, B. A., Leslie, J. F., Liew, E. C., Laurence, M. H., Bullock, S., Petrovic, T., Bentley, A. R., Howard, C. G., Peterson, S. A., \& Walsh, J. L. (2011). Fusarium species associated with plants in Australia. Fungal Diversity, 46(1), 1-27. 
Talhinhas, P., Batista, D., Diniz, I., Vieira, A., Silva, D. N., Loureiro, A., Tavares, S., Pereira, A. P., Azinheira, H. G., \& Guerra-Guimarães, L. (2017). The coffee leaf rust pathogen Hemileia vastatrix: One and a half centuries around the tropics. Molecular plant pathology, 18(8), 10391051.

(Tode)

L. Lombard

\& $\quad$ Crous.

(2016).

Mycobank. https://www.mycobank.org/page/Name\%20details\%20page/name/Paramyrothecium\%20roridu m

Torres, E. G., Cárdenas, J. L., Nieto, D. D. C., \& Soto, F. C. (2019). Experimento con biol de subproductos de azúcar para mayor rendimiento ecológico en el cultivo de lechuga (Lactuca sativa L.). Studium Veritatis, 17(23), 285-304.

Trakunyingcharoen, T., Lombard, L., Groenewald, J. Z., Cheewangkoon, R., To-Anun, C., \& Crous, P. W. (2015). Caulicolous Botryosphaeriales from Thailand. Persoonia: Molecular Phylogeny and Evolution of Fungi, 34, 87. https://doi.org/10.3767/003158515X685841

Vichi, F. F. (2015). La producción de café en México: Ventana de oportunidad para el sector agrícola de Chiapas. Espacio I+ D: Innovación más Desarrollo, 4(7). https://doi.org/10.31644/IMASD.7.2015.a07

Weir, B. S., Johnston, P. R., \& Damm, U. (2012). The Colletotrichum gloeosporioides species complex. Studies in mycology, 73, 115-180. 